To incorporate the features hoped for, there has been devised at the North Reading State Sanatorium a "Work Record" such as is presented herewith, and concerning which a few words may be given in brief explanation.

Under the first column heading, "Date," is given the day of the month of the conference and the dates of beginning, continuing or ending a certain line of exercise. Then columns for the description of the exercise prescribed, and the number of hours. In the weight column is given first the patient's usual weight before illness, his weight on admission, and opposite the date of conference his weight at that time. The "Cough and Expectoration" column provides for a description of the kind and amount of cough, with its increase or decrease, and the number of ounces sputum per twenty-four hours. The standard terms of use in the "Appetite" column are " excellent," "good," "fair" and "poor"; the same applying in the next column, "Digestion." Under "Malaise" is stated the presence, if any, of the various symptoms recognized by that general term, and their increase or subsidence between conferences.

Any extraordinary dyspnea is noted in the next to the last column, while the last column is left for "Remarks," under which a serious change in bi-daily temperature or pulse curve is immediately noted in red. However, this happens quite rarely, for the earlier signs properly observed and their significance noted indicate precautions which, if taken, preclude the occurrence of temperature elevation due to overexertion. In making use of the record chart careful questioning of the patient at conference time is necessary, followed by consideration of the data noted, whereupon the physician may decide what shall be the future course of patient's exercise, and note accordingly under " Exercise." This system has been attempted since the first of the new year at the North Reading State Sanatorium, and while there is not yet sufficient accumulation of data upon which to draw definite conclusions, the immediate results have nevertheless been so satisfactory as to seem to justify a thorough trial of the method. so favorable that we were led to experiment along the same lines to see if we could get the same results. The test as described by Russo gives a positive reaction in typhoid fever, measles, smallpox and sometimes in tuberculosis. It is performed by merely adding four drops of a one tenth of one per cent aqueous solution of methylene blue to four or five cubic centimeters of the patient's urine. The positive test is manifested by the urine, after thorough shaking, appearing green by transmitted light. It was claimed that boiling or the ingestion of such drugs as calomel, quinine, salol or caffeine would not affect the test. It was also said that as the disease advanced a returning blue color was a rather favorable prognostic sign.

Rolph and Nelson tried the test out on fifteen patients within forty-eight hours of entrance to the Toronto General Hospital. Thirteen of these gave a positive reaction, of which eight were negative to diazo and seven to Widal. The other two cases (of the fifteen) reacted negatively to all three of the tests. Of these, one gave a positive blood culture.

They also found that if bilirubin was present in the urine it gave a green color that was so nearly like the positive Russo that the urine would have to be discarded; also that the earlier in the disease the urine was examined, the more typical the reaction. The reason for the test was not found out, but it was supposed that it was a reduction test. They did not find that the return of the blue color was of any prognostic value.

The simplicity of the test, being such that it would be very efficacious in the hands of the general practitioner providing the results could be absolutely depended upon, led us to try it not only in cases of typhoid fever, but also in other diseases and in health. For this purpose samples of urine were collected from cases giving a positive Widal reaction at the Iowa State Board of Health laboratory, from cases in the University Hospital, and from the medical students of the university. In all, 118 samples were examined. The following table shows the results obtained.

NORTH READING STATE SANATORIUM.

Name

WORK RECORD.

Case No.

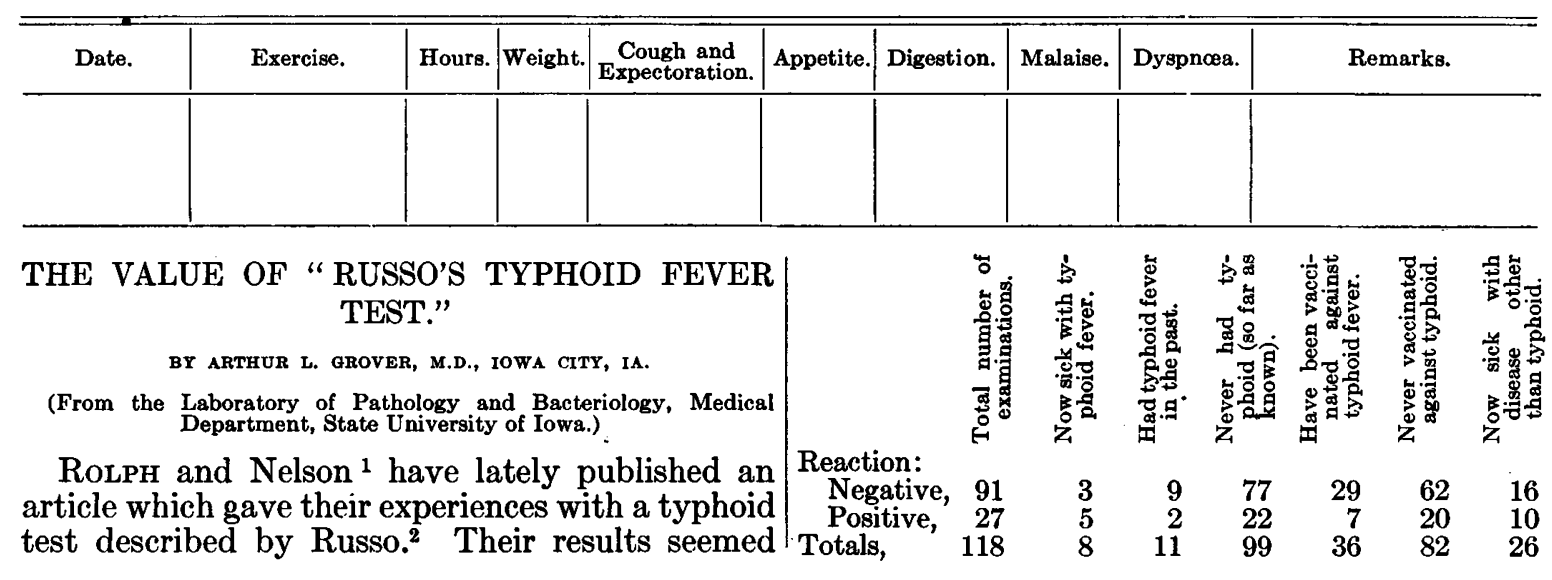


From the table it will be seen that our series consists of 118 samples of urine, of which 8 were taken from patients affected with typhoid fever, 26 were affected with other diseases, and the remaining 84 were taken from what seemed to be normal individuals. One of the eight typhoid cases was negative in the first week but became positive at the same time the Widal was positive. We called this positive in the table. One other gave a positive blood culture but a negative "Russo." The other six were in the second week of the disease.

The fact that eleven had previously had typhoid fever did not seem to have any particular bearing on the question. The proportion of positives in those who had never had typhoid fever was about the same as in those who had previously had it. Nor does vaccination against typhoid fever seem to have any particular bearing on the question.

Of the ten persons who gave positive Russo tests and who were affected by a disease other than typhoid fever, we found as hospital diagnoses: Chronic trachoma, carcinoma of superior maxilla, rhinitis sicca, appendectomy, deflected septum, depression of skull, dentigerous cyst, prolapsed uterus with endometritis, breaking adhesions of left maxilla, and one not given.

Of the sixteen negatives of those affected by some disease other than typhoid fever, we got as many different diagnoses as in the ten positive, so that nothing is to be gained from them. Only one of the positive urines gave any trace of bile.

On comparison, one urine from a student in perfect health at the time and since (two months) who had never had typhoid or been vaccinated could not be distinguished in color from one taken from a typhoid fever patient and reacting positive to the test.

Our conclusions are that although the test may be demonstrated in the urine of a large proportion of the typhoid fever cases, yet it may be demonstrated in such a large proportion of the urine of normal individuals that it is not a test that has any specific meaning or value as to the presence or absence of typhoid fever. When compared with the reliability of a blood culture it is worthless.

REFERENCES,

1 Some Experiences with " Russo's Typhoid Fever Test," by F. W. Rolph, A.M., M.D., Toronto, Ont., and W. Harper Nelson, M.B. Toronto, Ont. Med. Rec., Aug. 19, 1911

2 Russo, in Riforma Medica. Quoted by Rolph and Nelson.

\section{getedical Brogreg}

PROGRESS IN INTERNAL MEDICINE: DISEASES OF THE HEART.

BY FRANCIS W. PALFREY, M.D., BOSTON.

(Concluded from No. 18, p. 671.)

SECONDARY HEART DISORDERS AND NEUROSES.

SYMPTOMS from the physical crowding of the heart whether normal or enlarged have been discussed by Herz ${ }^{157}$ and others. These symp- toms are said to occur especially from faulty habitual positions, from deformities of the chest, or from influences causing high position or deficient mobility of the diaphragm, as in obesity or distention of the stomach.

Herz ${ }^{158}$ has attempted to describe individual groups of cases among the confused variety of patients with complaints commonly called cardiac neuroses. He distinguishes three types which seem to present definite symptom-complexes as follows: (1). Phrenocardia, occurring mostly in women and characterized by palpitation, pain in the region of the left nipple and a peculiar sighing or gasping inspiration attributed to hypertonicity of the diaphragm; unsatisfied sexual desire is set down as its chief or only cause, although this is disputed by Erb ${ }^{159}$ and by Treupel..$^{160}$ (2) Hypotonic bradycardia, characterized by abnormally low pulse-rate and subnormal blood pressure, without cause in myocardial insufficiency, with asthenia as the chief subjective manifestation. (3) Cardiac hypochondriasis, where excessive self-observation has led to the conviction that heart disease is present. The last two types occur most commonly in men. Erb and also Treupel guardedly confirm the existence of the symptom-complex of phrenocardia, but question its sexual origin. Berman ${ }^{161}$ and Morichan-Beauchant ${ }^{162}$ are more fully in agreement with Herz's views.

\section{TREATMENT.}

The numerous articles dealing with the treatment of heart disease may be divided into two classes, - those discussing the actions of drugs, and those describing dietetic and mechanical methods. The first class has to do chiefly with digitalis and its derivatives and allies. The second class is dominated by hydrotherapeutic treatment.

The action of digitalis on normal and on diseased hearts has been the subject of somewhat startling conclusions. Mackenzıe, ${ }^{4}$ in his book, has expressed the opinion based on extensive clinical observation that the benefit derived from digitalis is constant and striking only in cases of absolute arrhythmia, and that it is on account of its effect in these cases that its general reputation in the treatment of heart disease has been won. Cushny, ${ }^{140}$ whose authority on pharmacology is second to none, reports observations on patients in Mackenzie's clinic, and confirms the opinion of Mackenzie. He studied cases divided into two groups, the first comprising cases in which the dominant rhythm was normal, and the second composed of cases of auricular fibrillation or absolute arrhythmia, considering the effect of digitalis both in its action of producing inhibition by stimulation of the vagus nerve and also effects of direct muscular action. In the first class he found evidence of the vagus action in a diminution of rate in $30 \%$, and inferred a direct muscular action in some instances. In the second class, in contrast, he found slowing in $90 \%$. He suggests that the striking benefit in these cases of auricular fibril- 